\title{
Structural Variation in Surface Supported Synthesis by Adjusting Stoichiometric Ratio of the Reactants
}

Zhongmiao Gong,,$^{+\perp}$ Biao Yang, ${ }^{\dagger}{ }^{\perp}$ Haiping Lin, ${ }^{+,}$Yunyu Tang, ${ }^{+}$Zeyuan Tang,,$^{\dagger}$ Junjie

Zhang, ${ }^{\dagger}$ Haiming Zhang, ${ }^{\dagger}$ Youyong Li, ${ }^{\dagger}$ Yongshu Xie, ${ }^{*}$ Qing Li ${ }^{*}{ }^{* \dagger}$ Lifeng Chi ${ }^{*},{ }^{\dagger}$

${ }^{\dagger}$ Institute of Functional Nano \& Soft Materials (FUNSOM), Jiangsu Key Laboratory for Carbon-Based Functional Materials \& Devices, Soochow University, 199 Ren'ai Road, Suzhou, 215123, Jiangsu, P. R. China. "Key Laboratory for Advanced Materials and Institute of Fine Chemicals, East China University of Science and Technology, Shanghai 200237, P. R. China.

${ }^{\perp}$ These authors contribute equally to this work.

\section{Self-assembly of TFPB and TAPB monomers.}

We designed two precursors, the TFPB and TAPB, both of which have three-fold symmetry. Upon co-deposition of the two constitute molecules onto the $\mathrm{Au}(111)$ surface held at room temperature and carefully adjust the stoichiometric ratio to $1: 1$, the molecules self-assemble into a highly ordered phase, as shown in Figure S1a. The two components have similar size and shape, make them difficult to be identified. However, $d I / d V$ image, which reveals the different density of state at certain energy, clearly verifies a 1:1 stoichiometric ratio of the two precursors (Figure S1b). After annealing such a sample at $400 \mathrm{~K}, 2 \mathrm{D}$ frameworks are constructed as expected (Figure 
S1c). This product resembles to graphene, excepted for that " $A$ " sub-lattice of the structure is replaced with TFPB, whereas "B" sub-lattice is substituted with TAPB (the corresponding structural model is shown in Figure S1d, in which the black triangle represents TFPB molecule, and the red triangle represents TAPB molecule). Note that the amount of TFPB and TAPB is the same in the hexagonal network, suggesting that such a graphene-like framework is a result of the 1:1 composition ratio of the precursor compounds.
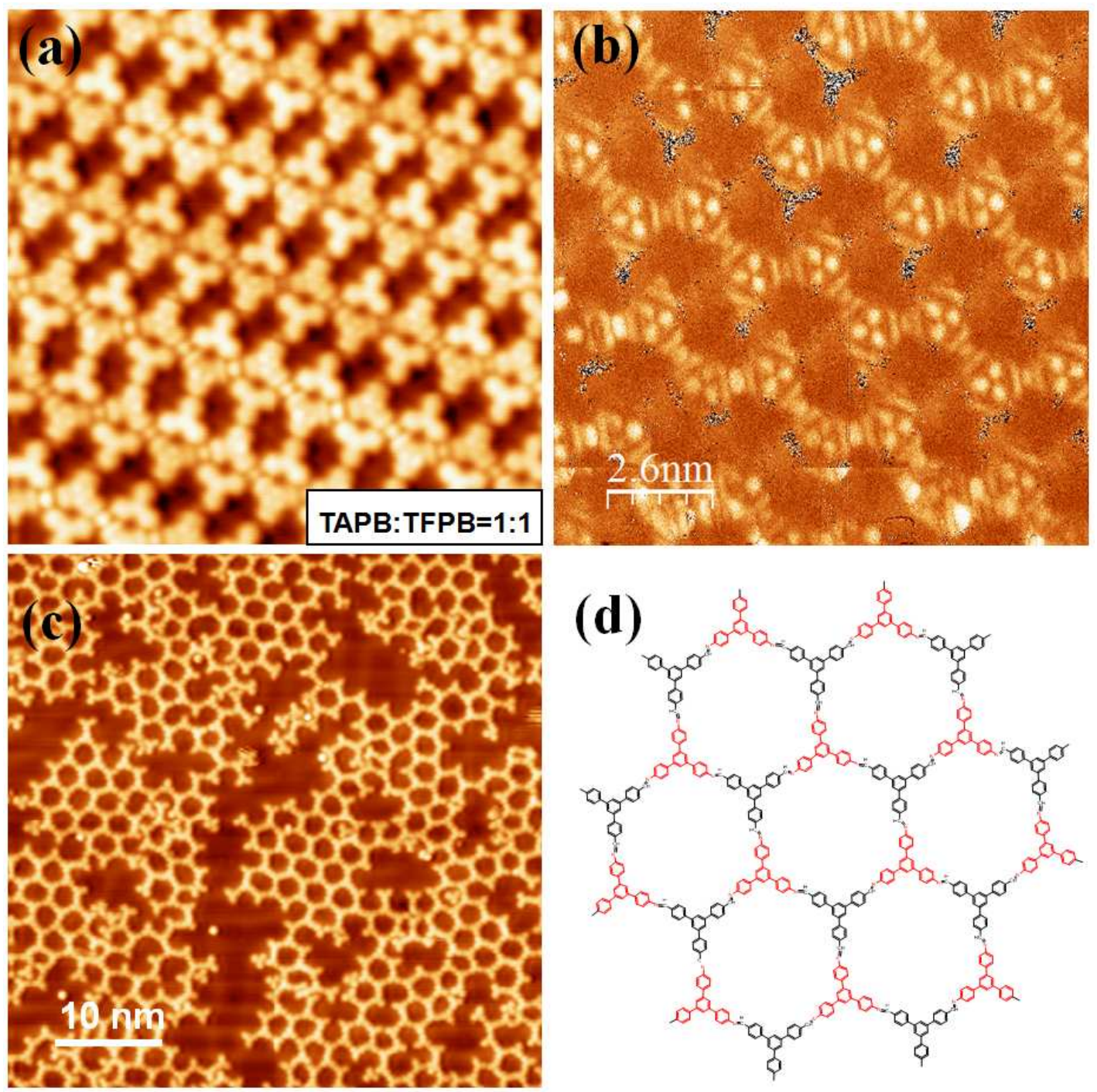

Figure S1. (a) STM image of the self-assembly phase by co-depositing 
1:1 TFPB and TAPB on the $\operatorname{Au}(111)$ held at room temperature. The superposed model represents the TFPB and TAPB monomers, respectively. (b) The simultaneously acquired $d I / d V$ mapping with $V_{\mathrm{b}}$ $=-1.2 \mathrm{~V}$. (c) STM image after annealing the self-assembled sample to 400 K for 20 minutes. (d) Structural model of (c).

\section{2. ${ }^{1} \mathrm{H}$ NMR spectrum of TFPB.}

The ${ }^{1} \mathrm{H}$ NMR spectrum of 1, 3, 5-tris(4-formylphenyl)-benzene (TFPB) is shown in Figure S5, which matches well with that previously reported reviously. ${ }^{1}$
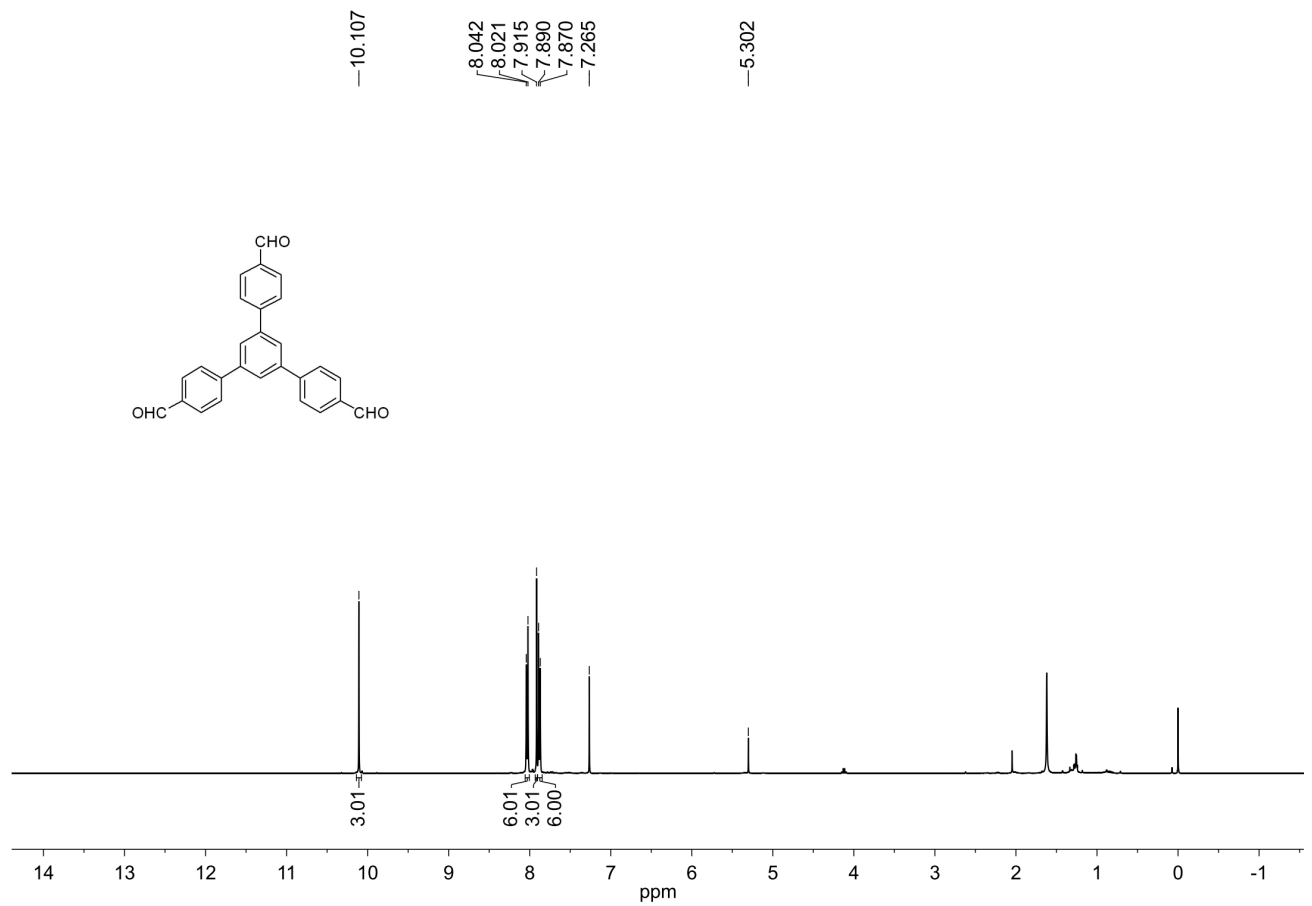

Figure S2. ${ }^{1} \mathrm{H}$ NMR spectrum of TFPB.

\section{References:}


1. Zhao, Y. C.; Wang, T.; Zhang, L. M.; Cui, Y.; Han, B. H. Facile Approach to Preparing Microporous Organic Polymers through Benzoin Condensation. ACS Appl. Mater. Interfaces 2012, 4, 6975-6981. 